\title{
Un análisis criminológico de la victimización de las personas sin hogar en la ciudad de São Paulo
}

\author{
Uma análise criminológica da vitimização das pessoas em situação de rua \\ na cidade de São Paulo \\ A criminological analysis of the victimization of homeless people \\ in the City of São Paulo
}

AUTOR

\section{Isabel García} Domínguez*

isabelgarcia

dominguez@usal.es

* Doctoranda en Estado de Derecho y Gobernanza Global en la USAL.

\section{RESUMEN:}

La criminalización de la pobreza y la selectividad del sistema penal, características del actual gobierno brasileño, han afectado, especialmente, al colectivo de personas sin hogar, cuyo aumento en la ciudad de São Paulo es una constante. Asimismo, la discriminación y la victimización penal, principalmente violenta, es una dinámica experimentada por estos sujetos durante los últimos años en la metrópoli mencionada. Con relación a lo expresado, las tipologías delictivas que más sufren son las agresiones físicas y/o verbales, unidos a los robos y/o hurtos, estableciéndose los porcentajes más bajos en la violencia sexual. Además, estos delitos son cometidos por diferentes autores, entre los cuales se destacan las fuerzas policiales. Igualmente, diversos estudios han sostenido que la motivación de la violencia que sufren las personas en situación de sinhogarismo se haya en el fenómeno aporófobo. En consecuencia, es necesario emprender una lucha contra la victimización penal que se ejerce en el colectivo de personas sin hogar de la ciudad de São Paulo.

\section{RESUMO:}

A criminalização da pobreza e a seletividade do aparelho punitivo, características do atual governo brasileiro, têm afetado especialmente o grupo de pessoas em situação de rua, cujo aumento na cidade de São Paulo é uma constante. Igualmente, a discriminação e a vitimização criminal, principalmente a violenta, é uma dinâmica vivenciada por esses sujeitos nos últimos anos na metrópole mencionada. Com relação ao expressado, as tipologias criminais que mais sofrem são as agressões físicas e/ou verbais, juntamente com assaltos e/ou furtos, ficando as menores percentagens para os crimes de violência sexual. Além disso, estes crimes são efetuados por diferentes autores, entre os quais se destacam as forças policiais. Da mesma forma, vários estudos têm argumentado que a motivação para a violência sofrida pelas pessoas em situação de rua está no fenômeno aporofóbico. Em consequência, é necessária uma luta contra a vitimização criminal sofrida pelo grupo de pessoas em situação de rua da cidade de São Paulo.

\section{ABSTRACT:}

The criminalization of poverty and the selectivity of the penal system are characteristics of the current Brazilian government. These phenomena have especially affected homeless people, whose increase is constant in the city of São Paulo. Likewise, discrimination and criminal victimization, mainly violent, is a dynamic experienced by these subjects in recent years in the aforementioned metropolis. In relation to what has been stated, the most suffered criminal typologies are physical and/or verbal aggressions, 
together with robberies and/or thefts, with the lowest percentages for crimes of sexual violence. Besides, these crimes are committed by different perpetrators, among which, the police forces stand out. In the same way, studies have argued that the motivation for violence suffered by homeless people resides in the aporophobic phenomenon. Consequently, it is necessary to fight against the criminal victimization suffered by the group of homeless people in the city of São Paulo. 


\section{Una aproximación al contexto político-criminal de Brasil: particular énfasis a la selectividad del sistema penal en el colectivo de personas sin hogar}

A seletividade penal se manifesta quando as instituições do sistema de justiça realizam constrangimentos e seleções para certos atores sociais, gerando desigualdades de tratamento no campo da segurança pública e da justiça criminal (Silvestre, Schilittler \& Sinhoretto, 2015, p. 3).

A partir de la segunda mitad de la década de los 90, Brasil experimentó un crecimiento de la crisis de la seguridad pública, entre otros factores, por los altos niveles de inseguridad, enmarcados en el punitivismo que ha sufrido la política criminal en América del Sur, asumiendo el gobierno un protagonismo mayor en las políticas criminales con el fin de reducir la violencia (Cifali, 2016).

En este sentido, bajo la justificación de otorgar seguridad -concepto que ha ocupado un lugar privilegiado en las políticas públicas-, se ha establecido un modelo de control del crimen militarista y encarcelador que se materializa con represión y cárcel, especialmente, en los delitos contra el patrimonio y los relativos al tráfico de drogas. Por lo tanto, la problemática se derivó de la utilización del Derecho penal como medio preferencial en la resolución de conflictos sociales, lo cual no sólo fue ineficaz, sino que empeoró la situación de los sujetos más desfavorecidos, como es el colectivo de personas sin hogar, a través de la criminalización de la pobreza ${ }^{1}$ y de la selectividad del sistema penal (Sinhoretto, Schilittler \& Silvestre, 2016).

Las políticas penales suelen focalizar su atención en los delitos cometidos por los individuos más vulnerables produciéndose una criminalización de la pobreza. Un ejemplo de lo referido es São Paulo -una de las cinco metrópolis más grandes del mundo- que poseía un $22 \%$ de la población penitenciaria del Estado de Brasil en el año 2013, con un 49,1\% condenados por delitos patrimoniales y un $25,3 \%$ por crímenes relacionados con las drogas, resultando, en la mayoría de las ocasiones, las detenciones de los sujetos más excluidos que cometen estos crímenes. En este sentido, la ley 11.343/2006 acrecentó la selectividad del sistema penal, sobre todo, en los sectores más desfavorecidos económicamente, así como la superpoblación carcelaria, debido a que elevó la pena mínima de los delitos de tráfico de sustancias estupefacientes y favoreció la interpretación de conductas de consumo propio como tráfico de drogas, penalizándolas ídem (Ghiringhelli y Cifali, 2016).

En la ciudad de São Paulo, la selectividad del sistema penal se puede observar en un informe publicado en el año 2016 que contabilizó unas tasas de detención de un 66,6\% en personas negras y un porcentaje de autoría del $86 \%$ de la Policía Militar, siendo esta el único testimonio de lo ocurrido en un $78 \%$ de los casos referidos, es decir, estableciéndose como la única prueba testimonial (cuyo peso es muy fuerte en el Estado brasileño), la cual, unida a la escasez de investigaciones, se consolida como la única prueba válida. En otras palabras, el testimonio de la policía equivale a la verdad judicial, incluso, cuando la violencia policial es corroborada en un $48,5 \%$ de los casos (siendo la Policía Militar responsable del 79,9\% de las agresiones), situándose por debajo la tasa de las amenazas proferidas, con un 40\% (Zaffalon, 2018). Además, conviene resaltar que para la mayor parte de los encarcelados pillados in fraganti no ha existido investigación, dinámica potenciada desde el año 2015 en el territorio brasileño a causa de la implantación de las audiencias de custodia por presiones internacionales.

Un ejemplo muy representativo de la criminalización de la pobreza y de la selectividad del sistema penal en el estado brasileño fue el caso de Rafael Braga, una persona negra de origen
PALABRAS CLAVE Sin hogar; victimización; violencia; sistema penal; São Paulo.

PALAVRAS-CHAVE Sem-teto; vitimização;

violência; sistema criminal; São Paulo.

KEYWORDS Homeless; victimization; violence; penal system; São Paulo.

Recibido: 09/07/2019

Aceptado: 20/10/2020 
humilde, usuario habitual de crack y en situación de sinhogarismo los años previos a su condena (Brandão, 2019).

El sujeto mencionado, una noche, en el contexto de la Copa Mundial de Fútbol de 2014, fue interceptado con dos garrafas, cuyo vertido eran productos que utilizaba para su trabajo, por la quinta Delegacia de Proteção à Criança e ao Adolescentes (DPCA). Este grupo policial, después de violentar a Rafael, lo acusó de transportar un coctel molotov con el fin de realizar un atentado terrorista en las manifestaciones que se estaban desarrollando, aunque los especialistas de la policía afirmaron con rotundidad que la mezcla de los productos contenidos en la garrafa no podía producir una bomba (Moura, 2018).

No obstante, Rafael Braga fue condenado a cinco años de prisión por el transporte de artefactos explosivos con la intención de fabricar un cóctel molotov para las manifestaciones expuestas previamente. Este evento, cambió su vida por completo, siendo una víctima más, no sólo del Derecho penal del enemigo², sino también de la selectividad del sistema penal racista y clasista (Moura, 2018).

Desafortunadamente, su relación con la justicia penal no se terminó con el suceso citado, sino que dos años después, cuando disfrutaba de su libertad provisional, fue víctima una vez más del sistema penal por supuesta posesión de drogas, a pesar de que Rafael alegó que fueron los policías quienes las colocaron con el fin de poder detenerlo. También, al igual que ocurrió en su primer contacto con el proceso de detención, sufrió violencia policial, e inclusive, amenazas de contenido sexual.

Finalmente, a pesar de la presentación de diversas pruebas por la defensa que negaban su autoría, el juez condenó a Rafael a más de 10 años de prisión, corroborándose lo manifestado por Kant de Lima (1997), esto es: los procedimientos judiciales brasileños persiguen el mantenimiento de un orden excluyente que criminaliza la pobreza en consonancia con la autoridad policial que ejerce una estrategia de sospecha sistemática ante los parias del sistema.

Por lo tanto, se puede observar: en primer lugar, la desproporcionalidad de la pena, condenándolo por tráfico de drogas, a pesar de la pequeña cantidad de estupefacientes poseída por el sujeto (en consonancia con la penalización de conductas que tienen cabida en el consumo propio expresadas previamente) y, en segundo lugar, la interpretación de "verdad absoluta" del testimonio de los policías. Con referencia a la última cuestión, esta se argumenta debido a que existió una insuficiencia de pruebas para su condena, el rechazo de estas mismas que favorecían su absolución y, por ende, la inaplicación del principio in dubio pro reo que debe primar en el establecimiento de una condena penal (Moura, 2018).

Asimismo, para los colectivos más desfavorecidos, como son las personas sin hogar, los tribunales no solo están inaplicando el principio de insignificancia penal, según la jurisprudencia del Supremo Tribunal Federal de Brasil, sino que están sancionando conductas de bagatela (Aguirre, 2018). Un ejemplo fue el hurto de unas bermudas avaladas en diez reales brasileños, lo que equivale, aproximadamente, a dos euros, a causa de que, el juez determinó que era una persona sin hogar, adicta a las drogas y que "vivía" cometiendo delitos patrimoniales, imponiéndole una pena de privación de libertad de un año y siete meses en régimen cerrado (Valente, 2018).

Con relación a la selectividad del sistema penal, existen leyes específicas que criminalizan a las personas sin hogar por ser "peligrosos", por ejemplo, la Ley n 11.983/2009 proferida por el Estado brasileño en el año 2009, que revocó la contravención del artículo 60 de la Lei de Contravenções penais, la cual preveía prisión simple, de 15 días a 3 meses para quien, y cito literalmente (traducido del portugués), "mendigara por ociosidad o cupidez" (Oliveira, 2018, p. 15).

Ahora bien, los procesos mencionados se engloban en el tránsito de la modernidad a la postmodernidad, cuyo efecto fue la transformación de una sociedad inclusiva de estabilidad (cuyos valores esenciales se configuran por la familia y el trabajo) a una sociedad excluyente de cambios y divisiones en los ámbitos: económico (enfatizando en el consumismo), social (con la consiguiente reducción de políticas sociales) y de 
justicia (concentrando sus acciones en la criminalización de la pobreza, como ya se ha explicitado) (Young, 2002).

En consecuencias, en estas nuevas sociedades, caracterizadas por la inseguridad ontológica ${ }^{3}$, existen colectivos potencialmente desviados, pertenecientes a sectores marginados, producto de las dinámicas del mercado y del individualismo, quienes adoptan una cultura de la exclusión que, como se ha demostrado, revierte en la criminalidad, ya sea en sus causas o en sus consecuencias (Young, 2002). De esta forma, los individuos que no tienen posesiones, es decir, que no tienen capacidad de consumo no son reconocidos como sujetos, y, además, son considerados peligrosos para la sociedad (Bertty \& Rodrigues, 2018). También, en la actualidad se están aplicando políticas de seguridad pública para el control de estas poblaciones "indeseadas" (Zaffalon, 2018).

Estas dinámicas, expuestas en los párrafos anteriores, han afectado en gran medida a un colectivo, las personas sin hogar, quienes sufren las medidas adoptadas por el gobierno para su invisibilización, acompañadas, en muchas ocasiones, de la aceptación de la sociedad. En efecto, en los últimos años se ha producido una elevación del colectivo de sinhogarismo, con la discriminación y vulnerabilidad que implican, con una cifra de 101 mil personas contabilizadas en esta situación en el año 2016 para todo el territorio brasileño, concentradas fuertemente en las ciudades más grandes (Carvalho, 2016). Asimismo, Zaffalon (2018) indica que el colectivo de sinhogarismo es víctima de extremismos y arbitrariedades de los agentes sociales y económicos más poderosos, y es que las investigaciones realizadas concluyen que el sistema brasileño es opresor e injusto, especialmente con la pequeña delincuencia (Kant de Lima, Misse \& Mendes, 2000).

Con base a todo lo aludido, el objetivo principal del presente artículo es realizar un estudio sobre la victimización penal de las personas sin hogar en la ciudad de São Paulo, quienes, de forma paralela, son criminalizados, como se presentará, brevemente, en los siguientes apartados.

\section{La victimización penal del colectivo de sinhogarismo en la ciudad de São Paulo}

\subsection{Aspectos generales: definición y causas del sinhogarismo}

En un enfoque tridimensional, se podría definir al sinhogarismo, es decir, a la falta de vivienda, como una forma de discriminación sistémica y de exclusión social, acompañada de una lucha por la supervivencia y la dignidad (Oliveira, 2018)4. Además, el concepto de persona sin hogar adoptado en el presente artículo es amplio, en consonancia con el criterio acogido en las investigaciones que serán expuestas a lo largo de este apartado, abarcando a las personas que de forma, temporal o permanente, pernoctan en espacios públicos de la ciudad, edificios abandonados (excluyendo a los okupa), albergues públicos y/o entidades sociales (Prefeitura Municipal de São Paulo, 2015).

En cuanto a las causas del sinhogarismo, algunos estudios realizados en 2007 ponen de manifiesto que las más habituales en la sociedad brasileña para desembocar en esta situación son: problemas con las drogas o el alcoholismo (35\%), el desempleo $(29,8 \%)$ y disputas familiares $(29,1 \%)$ (Centurião, Rodrigues, Teixeira, 2018), las cuales no han sufrido modificaciones en los años siguientes, según datos del 2015 (Ministerio de Saúde, 2019). En efecto, las primeras variables mencionadas son una constante en este colectivo, sobre todo, en el género masculino, con porcentajes tan abrumadores como el $70 \%$ de consumo de alcohol o el 52,5\% de drogas ilícitas para el grupo de individuos que se encuentra de forma permanente en la calle, siendo el porcentaje menor para los sujetos que pernoctan en albergues, con un $44,6 \%$ y un $28,7 \%$ respectivamente, de acuerdo a las estadísticas del año 2015 (Prefeitura Municipal de São Paulo, 2015b).

En las personas sin hogar, la pobreza extrema se relaciona con las drogas, entendida como un escape mental de su situación, aunque finalmente se convierte en una dinámica difícil de abandonar (Nascimento, 
Rodrígues \& Centurião, 2018), siendo cracolandia la máxima representación de lo mencionado en la ciudad de São Paulo.

Respecto a cracolandia, es un área de la región central de São Paulo, donde se concentran usuarios de crack en la calle para consumir esta droga, principalmente personas sin hogar (debido a que su consumo comienza con esta condición de forma generalizada), con una pérdida total -o parcial en el mejor de los casos- de sus relaciones afectivas y sin ningún sustento económico. El perfil habitual de este barrio es un hombre, poliusuario de drogas, que utiliza todo tipo de medios a su alcance, independientemente de su legalidad, para perpetuar su consumo. Asimismo, en estas áreas se desarrolla una cultura de resistencia frente a los valores sociales hegemónicos (Raupp \& Adorno, 2010).

En el abordaje del enfoque de género, su situación es aún más complicada debido a que sufren, inicialmente, una doble discriminación: por ser mujer y por su situación de sinhogarismo, la cual se puede convertir en triple, con la adhesión de la prostitución, que es un oficio recurrente en este colectivo (Teixeira, 2016).

\subsection{Un análisis de la evolución del sinhogarismo en la ciudad de São Paulo}

Con relación a la evolución del sinhogarismo, se puede observar un aumento progresivo en la ciudad de São Paulo. La cifra de este fenómeno se elevó desde $1991^{5}$ hasta $2019^{6}$ con un aumento porcentual en torno al 617\%, experimentando el último periodo, comprendido del 2015 al 2019, un crecimiento del 53\% (Prefeitura Municipal de São Paulo, 2020a), como presenta el siguiente gráfico:

Asimismo, el gráfico muestra la evolución ligeramente divergente entre personas que pernoctan en la calle y sujetos que lo hacen en instituciones sociales, experimentando estos últimos un aumento mayor en el periodo 20002009. De igual modo, cabe destacar que, en comparación con el crecimiento de la población de São Paulo, el porcentaje de personas en situación de sinhogarismo se elevó en mayor medida, con una variación del $0,7 \%$ de la primera en contraste con un $4,1 \%$ de la segunda (Prefeitura Municipal de São Paulo, 2015a).

En consideración a los datos expuestos en los párrafos anteriores, cabe enfatizar en el colectivo de personas que pernoctan en la calle que: la expansión se produjo más en zonas periféricas de la ciudad, la existencia de un conocimiento generalizado acerca de los centros de acogida, a los cuales recurren, ocasionalmente, un porcentaje alto de este colectivo, y el perfil mayoritario, constituido por un hombre (en un $84 \%$ de los casos), con una edad media de 39 años, de color de piel diferente al blanco (con un porcentaje del $73 \%$ ) y brasileño (con una tasa del $98,4 \%$, cercana al $100 \%$ ).

Por el contrario, los albergues contienen sujetos con una edad media más elevada, con 42 años, y poseen un porcentaje mayor de extranjeros, correspondiente al $7 \%$, siendo las demás características similares, a excepción del incremento progresivo que fue más acentuado en este grupo (Prefeitura Municipal de São Paulo, 2015a).

Acerca de la violencia ejercida hacia las personas sin hogar, la cual será desarrollada en los siguientes subapartados, cabe destacar que su aumento se ha producido en la sociedad brasileña de forma 
generalizada. Sin embargo, esta se reproduce de forma más intensa entre las personas sin hogar por la vulnerabilidad de su condición (Prefeitura Municipal de São Paulo, 2015a).

\subsection{El sinhogarismo en la ciudad de São Paulo (2010-2015): especial referencia a la victimización penal y a la autoría de la violencia ejercida}

A continuación, se expondrán los datos relativos al periodo 2010-2015 con el fin de analizar sus variaciones, aunque el estudio al cual se hará referencia tiene una limitación, consistente en estudiar únicamente el área central de la ciudad de São Paulo, dejando relegada la periferia de la metrópoli (Prefeitura Municipal de São Paulo, 2015d). Asimismo, se expondrán las diferencias más significativas entre dos subgrupos poblacionales: las personas $\sin$ hogar que duermen en albergues (categorizados en el grupo $A$ ) y las personas sin hogar que pernoctan en la calle (asignadas al grupo B).

En primer lugar, el perfil de persona sin hogar se modificó ligeramente, con un aumento del género masculino, las personas con una piel no blanca y la edad media. Igualmente, la última característica mencionada experimentó una variación, especialmente, en las personas que pernoctan en la vía pública (grupo B) con una diferencia entre uno y tres años en comparación con el grupo A. Con relación al consumo de drogas en el colectivo objeto de estudio también se elevó, en un $10 \%$, alcanzando a un $84 \%$ de esta población en 2015, con una manifiesta sobrerrepresentación de los sujetos que se ubican en el grupo B (Prefeitura Municipal de São Paulo, 2015d).

En segundo lugar, la discriminación se produjo de forma generalizada en los dos grupos, aunque el porcentaje fue mayor en el grupo B, con una diferencia porcentual respecto al grupo A superior al $10 \%$ en tiendas, bares y restaurantes. Haciendo alusión a la violencia, la dinámica de la victimización se repite, produciéndose esta de forma más acentuada en el grupo B, aunque se elevó en los dos grupos en comparación con el 2010, siendo los autores más relevantes: la policía y los transeúntes (Prefeitura Municipal de São Paulo, 2015d). El crecimiento mencionado, se puede observar en las diferentes categorías delictivas, a través del siguiente gráfico:

En efecto, a pesar del aumento progresivo de todos los crímenes mencionados, los robos y/o hurtos experimentaron un crecimiento mucho más acentuado, en consonancia con las agresiones verbales, situándose ambas con las cifras más altas en comparación con las tipologías restantes. Por otro lado, la violencia sexual y las agresiones físicas sufrieron en menor medida el incremento, obteniendo la primera de ellas, el menor porcentaje de todas las categorías estudiadas.

En tercer lugar, para ultimar la comparativa de los resultados obtenidos entre los años 2010 y 2015, también se contempló un aumento relevante en la criminalización de estas personas (corroborado por su estancia en centros penitenciarios) con una elevación del $13 \%$, alcanzando una tasa del $40 \%$ en el año 2015. Del mismo modo, existió una sobrerrepresentación de las personas jóvenes, del uso de drogas y de la tasa de victimización violenta, esta última, sobre todo, en contraste con el resto de la población. Además, se halló un porcentaje mayor de personas del grupo B que del grupo A para la categoría analizada (Prefeitura Municipal de São Paulo, 2015d). 
Continuando el análisis del año 2015, en el cual se han recolectado el mayor número de estadísticas sobre el objeto de estudio, es decir, la victimización penal de las personas sin hogar, las investigaciones pusieron de manifiesto 15905 personas sin hogar en la ciudad de São Paulo, situándose 8570 en servicios de acogida y 7335 en las calles. Por lo tanto, la diferencia del tamaño muestral es significativa y deberá ser considerada en la interpretación de los resultados (Prefeitura Municipal de São Paulo, 2015c).

En el análisis de la violencia (debido a que ya se han comentado las variaciones en el perfil de la persona sin hogar y el uso de drogas), una de las conductas discriminatorias que experimentó el colectivo de personas sin hogar en la ciudad de São Paulo fue el impedimento de la entrada o su expulsión de diferentes establecimientos, con una frecuencia mayor en bares y restaurantes, según datos del 2015. Sin embargo, un porcentaje referente al $3 \%$ de estos individuos, también sufrió esta situación en servicios de asistencia social, estableciéndose este como un comportamiento injustificable debido a que estos centros están destinados, entre otros colectivos específicos, a personas sin hogar, y deben prestarles una atención focalizada (Prefeitura Municipal de São Paulo, 2015c).

Prosiguiendo con la victimización del colectivo objeto de estudio, y centrando la atención en el grupo A (por ser su muestra más representativa), los datos muestran como el hurto y/o robo de sus pertenencias es el crimen que más padecen, con 4824 víctimas (que representa una tasa de victimización del 59,4\%), cuya diferencia porcentual con la agresión verbal es insignificante (con una tasa de victimización del 55\%), en la cual caben comentarios como humillaciones, ofensas o insultos. Continuando con la frecuencia, la violencia física es un patrón que se repite, cuya victimización se produjo en un $37,5 \%$ de la muestra. Asimismo, las conductas de tentativas de remoción forzada, homicidio con diferentes instrumentos, y violencia sexual obtuvieron porcentajes menores, sobre todo, esta última que no superó el $5 \%$ de victimización de la muestra (Prefeitura Municipal de São Paulo, 2015c).

Con relación a la cuestión de género, conviene resaltar que las mujeres, a pesar de su cifra menor en el conjunto de personas sin hogar y su edad más cercana a los 35 años, tienen que lidiar con diferentes situaciones de violencia diaria de forma más acentuada que los hombres (Prefeitura Municipal de São Paulo, 2015a).

En cuanto a los actores de la victimización explicada anteriormente, es decir, referente al grupo A, la agresión verbal se produjo, principalmente por transeúntes, policía militar (PM de aquí en adelante) y/o civil, personas sin hogar, Guardia Civil Metropolitana (GCM de aquí en adelante) y seguridad privada, citados en orden de su frecuencia. De forma similar, la agresión física fue perpetrada en la mayoría de las ocasiones por personas sin hogar, PM, policía civil y transeúntes. En la categoría de tentativa de homicidio, las diferencias porcentuales son más elevadas, aunque un $48,3 \%$ de la muestra sufrió la realización de esta conducta por personas sin hogar, un $27,5 \%$ por transeúntes y un $26,3 \%$ por la PM y/o civil (Prefeitura Municipal de São Paulo, 2015c). En este sentido, se debe destacar la inconcebible producción de estos actos por la policía, quien debería salvaguardar su seguridad, aunque, como se puede percibir, los patrones son diferentes de lo que cabría esperar. Por otra parte, los autores de los crímenes sexuales, robos y hurtos, fueron mayoritariamente personas sin hogar, siendo los transeúntes la segunda categoría de ambos, con una variación muy elevada en contraste con el resto de categorías. (Prefeitura Municipal de São Paulo, 2015c).

Ahora bien, independientemente de la comisión de estos crímenes por las personas sin hogar, sobre todo, en el ámbito patrimonial, donde este grupo obtuvo el porcentaje más elevado, con un $71,1 \%$ de la práctica de los delitos de robo y/o hurto sufridos por la muestra del grupo A, se debe señalar que las fuerzas policiales, en sus diferentes categorías, obtuvieron porcentajes significativos en todas las tipologías delictivas, lo que denota una de las problemáticas existentes en la ciudad de São Paulo (Prefeitura Municipal de São Paulo, 2015c).

Por lo que se refiere a las personas del grupo B (cuyo tamaño muestral fue menor), la discriminación sufrida se sitúa, principalmente, en restaurantes y bares de igual forma, aunque su porcentaje es más elevado. En alusión a la violencia, por orden de mayor padecimiento, ha sido: agresiones verbales, robos y/o hurtos, y 
agresiones físicas, con porcentajes superiores al $50 \%$, y más específicamente, con un $70 \%, 66.4 \%$ y $50.4 \%$ respectivamente. En otras palabras, más de la mitad de la muestra sufrió, al menos en una ocasión, los crímenes comentados (Prefeitura Municipal de São Paulo, 2015c).

De acuerdo a los agentes de la violencia del grupo B, para la categoría de agresión verbal destacan los transeúntes y la PM y/o civil, seguidos de las personas sin hogar, la GCM, los comerciantes y la seguridad privada. Igualmente, en las agresiones físicas, los autores son los mismos, aunque las personas sin hogar pasan a ser la primera categoría, duplicando al resto con un 63,5\%, a excepción de la PM y/o civil que se sitúa con un $36,2 \%$. En cuanto a la tentativa de homicidio, fue cometida principalmente por personas sin hogar, con una diferencia porcentual elevada respecto a las otras categorías, y por la PM y/o civil, como actores secundarios y terciarios, con unos porcentajes que oscilan en torno al $25 \%$. De igual modo, para los delitos sexuales y los robos y/o hurtos, el actor mayoritario es el colectivo de personas sin hogar, con un $53,3 \%$ y un $83,3 \%$ respectivamente, siendo los transeúntes la segunda categoría (Prefeitura Municipal de São Paulo, 2015c).

A continuación, se realizará una comparativa con relación a las variables estudiadas para los grupos A y B, comenzando con las tipologías de victimización expuestas en el siguiente gráfico:

La victimización es más elevada para el grupo $\mathrm{B}$ en todas las categorías delictivas, sobre todo si tenemos en cuenta que la muestra fue mayor para el grupo A, por lo que la mayor exposición al riesgo que experimenta el grupo de personas que pernocta en la calle se puede observar en las investigaciones realizadas. Por otro lado, ambos grupos coinciden en el orden de frecuencia de las tipologías sufridas, encontrándose la mayor diferencia en las agresiones verbales y físicas (con una diferencia porcentual de 14,8\% y $12,9 \%$ respectivamente), y el menor contraste entre los grupos en los crímenes sexuales (con un $1,5 \%$ de variación).

Relacionado con la autoría de las victimizaciones, en ambos grupos la tentativa de homicidio fue cometida principalmente por personas sin hogar, con una diferencia porcentual elevada en comparación con las otras categorías, y por la PM y/o civil, como actores secundarios y terciarios. Igualmente, para los delitos sexuales y los robos y/o hurtos, el actor mayoritario es el colectivo de personas sin hogar, con un $50 \%$ aproximadamente en los grupos A y B para el primer delito, y un $71,10 \%$ del grupo $\mathrm{A}$ en comparación al $83,3 \%$ del grupo B para el segundo delito, siendo los transeúntes la segunda categoría de sendos crímenes.

En suma, el colectivo de sinhogarismo victimiza a las personas que se encuentran en su misma condición sin diferencias significativas entre el grupo A y el grupo B, a excepción de la tipología de robos/hurtos, como se muestra en el gráfico: (Gráfico 4).

Para ultimar la comparativa (observar el gráfico que se presenta a continuación para una mayor comprensión), cabe destacar que las fuerzas policiales, en sus diferentes categorías, obtuvieron porcentajes significativos en todas las tipologías delictivas, para sendos grupos. En estos, las diferencias se muestran en dos delitos: la agresión verbal y el abuso/violencia sexual, las cuales son cometidas en porcentajes más elevados en el grupo B, en especial, en el segundo delito, reiterándose la mayor exposición al riesgo de este colectivo, ya comentada (Prefeitura Municipal de São Paulo, 2015c). Por lo tanto, se ha otorgado visibilidad a la 
victimización que ejerce la policía en el colectivo de sinhogarismo (Gráfico 5).

\subsection{El sinhogarismo en la ciudad de São} Paulo desde el año 2015 hasta la actualidad: particular mención al fenómeno aporófobo

En los años siguientes, no existen investigaciones realizadas por organismos oficiales que se refieran a la victimización penal, con sus correspondientes tipologías, que padece el colectivo de personas sin hogar en la ciudad de São Paulo. Sin embargo, un estudio recabó durante los años 2015, 2016 y 2017 las notificaciones de violencia experimentadas por este colectivo en el Sistema de Informação de Agravos de Notificação (Sinan) que forma parte del Sistema Único de Saúde del territorio brasileño. Los resultados fueron 777.904 casos de violencia, cuyas comunicaciones eran más frecuentes por: jóvenes (con referencia a la edad), mujeres (según el género) y personas de color (según la raza) (Ministerio de Saúde, 2019). Además, haciendo alusión a la geografía, São Paulo presentó el mayor número de notificaciones de violencia.

A proposito de la violencia, la física fue mayoritaria, siendo experimentada por un 92,9\% de las personas analizadas, situándose seguidamente la psicológica con un 22,2\% y la sexual, con un porcentaje más reducido, con un $3,9 \%$. Adicionalmente, los autores de esta violencia fueron desconocidos en la mayor parte de las notificaciones (referentes a un $37 \%$ ), con un porcentaje menor de conocidos (con un $33,7 \%$ ) y obteniendo la policía un 4,2\% (Ministerio de Saúde, 2019).
Las personas sin hogar como autores de las victimizaciones al colectivo de personas sin hogar de la ciudad de São Paulo (2015)

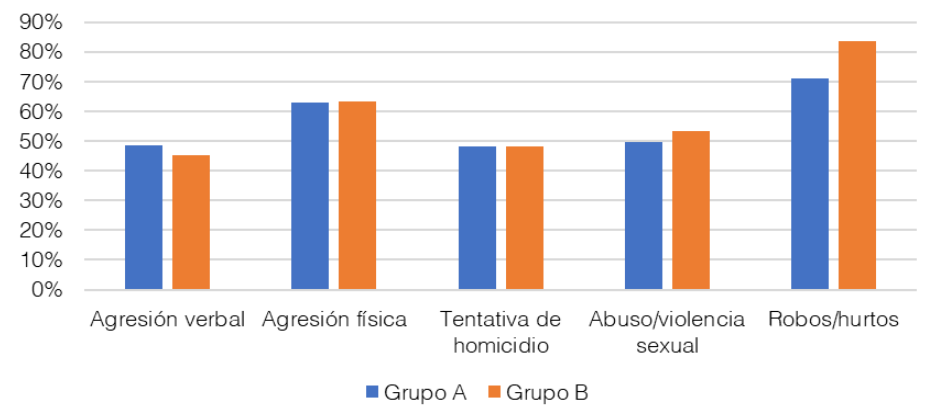

Gráfico 4. Las personas sin hogar como autores de las victimizaciones al colectivo de personas sin hogar de la ciudad de São Paulo (2015) con base a los resultados de la investigación efectuada por la Prefeitura Municipal de São Paulo (2015a; 2015c).

Porcentaje de autoría de la Policía civil y/o militar en las victimizaciones al colectivo de personas sin hogar de la ciudad de São Paulo (2015)

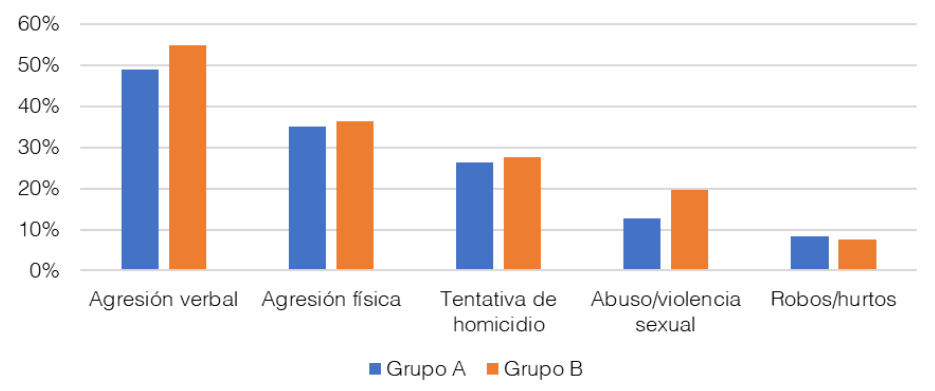

Gráfico 5. Porcentaje de autoría de la Policía civil y/o militar en las victimizaciones al colectivo de personas sin hogar de la ciudad de São Paulo (2015) a partir de los datos obtenidos a través de la Prefeitura Municipal de São Paulo (2015c).

Por lo tanto, existe una contradicción entre las estadísticas presentadas por la Prefeitura Municipal de São Paulo en sus diferentes años y el Ministério de Saúde, debido a que, de acuerdo con los datos recabados por el primer organismo citado, el autor de la violencia es constituido, principalmente, por la policía, a diferencia del segundo que atribuye como responsable mayoritario a personas desconocidas, con un porcentaje insignificante a las fuerzas policiales.

Una explicación plausible de la incongruencia presentada es que los sujetos que acuden a los servicios de salud tengan miedo de confesar que la policía fue el ejecutor de la violencia, ya sea por las repercusiones que puede conllevar, o el temor subjetivo a estas. De igual modo, otra hipótesis podría ser que las personas que son víctimas de violencia ejercida por la policía no acuden a los centros médicos y/o hospitales, o, inclusive, que la policía no sea un actor de la violencia perpetrada contra estas personas.

No obstante, en la última explicación existe una dificultosa credibilidad, al menos observando los estudios expuestos previamente, los cuales no se realizan en instituciones oficiales (a pesar de ser realizado por un 
organismo oficial), lo que, habitualmente, otorga más confianza y seguridad a las personas sin hogar para narrar sus victimizaciones.

Con relación a las hipótesis presentadas, el Centro Nacional de Defesa de Direitos Humanos (DCNDDH) indicó que en el año 2017 se registraron, en un 65\% de las denuncias de violencia sobre personas en situación de sinhogarismo, que el Estado había sido el actor, constituyéndose este como el principal agente violador de los derechos humanos de este colectivo (Schramm, 2017), lo que descartaría, inicialmente, la afirmación de que la policía no se configura como el autor mayoritario de la violencia que sufren estas personas. Sin embargo, ante la falta de estudios más profundos acerca de esta temática, solo se puede realizar una aproximación, quedando pendiente de ser investigada con el fin de poder concluir con rigor científico cual es la realidad social actual.

Ahora bien, continuando con el estudio presentado, una cuestión muy importante que trata, a diferencia de las investigaciones expuestas anteriormente, es la motivación de la violencia, y más específicamente, de la motivación aporófoba. En efecto, el Ministerio de Saúde (2019) contabilizó 17.386 casos violentos con motivación aporófoba, es decir, por su situación de extrema pobreza manifestada a través del sinhogarismo. Esta, para el periodo de estudio, se produjo en un 20,1\% en la ciudad de São Paulo, registrando el sudeste un $54 \%$ de las notificaciones aporófobas.

La aporofobia ${ }^{7}$, definida como el odio, desprecio o rechazo hacia los pobres, afecta diariamente al colectivo de personas sin hogar a causa de su condición de extrema vulnerabilidad y exclusión social. No obstante, este fenómeno, caracterizado por la escasez de investigaciones y datos, ha experimentado un auge en su estudio en los últimos años (García, 2020), sobre todo, en el territorio español, a través de investigaciones como la realizada por el Observatorio Hatento (2015) en diferentes ciudades españolas, organismo que concluyó una tasa de victimización del 47,1\% para la muestra de personas sin hogar estudiada, u otras más focalizadas, como la realizada en la ciudad de Salamanca, que nos ofrece una tasa de victimización del $75 \%$, con un tamaño muestral inferior (García, 2019).

En alusión a la ciudad de São Paulo y a la cuestión de género, una de las victimizaciones de corte aporófobo que se han observado para el colectivo de mujeres sin hogar, y más específicamente de aporofobia institucional, es la esterilización de estas (sin su consentimiento) por orden judicial, con la correspondiente violación de los derechos que implica, como la dignidad humana (Mello, 2018). Asimismo, en las prácticas criminalizadoras y selectivas ejercidas por el sistema penal, que han sido abordadas, brevemente, en el primer apartado de este trabajo, también se observa el fenómeno aporófobo. Por ejemplo, en la penalización de conductas de bagatela, como el hurto de unas bermudas cuyo valor fue insignificante.

Por lo tanto, ante la ausencia de estudios sobre criminalización y victimización por razón aporófoba en la ciudad de São Paulo, la autora desarrolló dos investigaciones empíricas para comprobar su existencia en el territorio mencionado, las cuales se desarrollaron de forma paralela en las audiencias de custodia del Fórum Criminal de Barra Funda ${ }^{8}$, y con el Movimento Nacional da População de Rua ${ }^{9}$. Sin embargo, estas se encuentran en período de análisis, aunque la autora adelantó que la aporofobia es un fenómeno presente en el colectivo de sinhogarismo de la ciudad de São Paulo.

La conclusión adelantada previamente ya ha sido manifestada por diversos autores, como Aguirre que expuso:

o ser humano é naturalmente "aporófobo", possui mecanismo dissociativo que Ihe afasta de acontecimentos e pessoas desagradáveis. As instituições, porque compostas por indivíduos, reproduzem a tendência humana de segregar os diferentes e rechaçar os indesejáveis (Aguirre, 2018, p. 458). 
cuya traducción es:

el ser humano es naturalmente aporófobo, posee un mecanismo disociativo que le aleja de acontecimiento y personas desagradables. Las instituciones, compuestas por individuos, reproducen la tendencia humana de segregar a los diferentes y rechazar a los indeseables.

Para finalizar, con relación al estudio más reciente sobre el sinhogarismo, este data del año 2019, cuya contabilización fue de 24.344 personas sin hogar, perteneciendo 11.693 al grupo A y 12.651 al grupo B (Prefeitura Municipal de São Paulo, 2020a). En este, se abordó el perfil de persona sin hogar, configurado por un hombre (en un $85 \%$ de los casos), con una media en torno a los 41 años (siendo la categoría mayoritaria entre los 31 y 49 años) y con piel diferente a la blanca (en un $78 \%$ de los casos). De igual forma, las diferencias entre el grupo A y B fueron insignificantes, obteniendo el grupo B una media de edad menor (Prefeitura Municipal de São Paulo, 2020b), al igual que mostró la investigación del 2015. Desafortunadamente, este censo no recogió datos acerca de la discriminación u otras formas de violencia sufridas por el grupo de personas sin hogar.

\section{Conclusión}

En consonancia con la sociedad excluyente a la que se asiste, la criminalización de la pobreza y la selectividad del sistema penal en el gobierno brasileño han repercutido fundamentalmente en los sujetos más desfavorecidos, especialmente en las personas sin hogar, concentrando la atención en los delitos patrimoniales y los relativos al tráfico de drogas, como muestran las estadísticas penitenciarias. Asimismo, el aparato policial y los juzgados son partícipes de estas dinámicas, poniendo de relieve la violencia que habitualmente ejerce la Policía Militar en sus detenciones.

Con relación al grupo mencionado en el párrafo anterior, el sinhogarismo, definido como la falta de vivienda, supera los 100.000 individuos en el territorio brasileño, experimentando la ciudad de São Paulo un aumento progresivo en los últimos 30 años, especialmente para el periodo del 2015 al 2019. Las causas más frecuentes para desembocar en esta situación fueron las drogas, el alcohol, el desempleo y los problemas familiares, sin la existencia de modificaciones representativas en los periodos temporales analizados. De igual modo, el perfil de persona sin hogar, constituido por un hombre, no blanco, con una medida de edad de 40 años y consumidor de alcohol y/o drogas, no sufrió variaciones significativas en los años analizados.

Respecto a la victimización de las personas sin hogar, comenzando con la discriminación, esta es una dinámica constante que experimenta el colectivo objeto de estudio, sobre todo en bares y restaurantes. Ahora bien, en alusión al ámbito penal, este grupo ha sufrido, en orden de una mayor frecuencia: agresiones físicas y verbales, robos/hurtos y violencia sexual, obteniendo el subgrupo de sujetos que pernoctan en la calle una frecuencia superior en la victimización por la especial vulnerabilidad que es causa de su constante exposición al riesgo.

Haciendo alusión a la autoría de las victimizaciones, las personas sin hogar y las fuerzas policiales se consolidaron como los principales perpetradores, con variaciones porcentuales en función de las tipologías delictivas. No obstante, conviene resaltar que las fuerzas policiales obtuvieron porcentajes elevados en todas las categorías delictivas, siendo esta cuestión afirmada por diversos estudios, aunque otras fuentes designan a los desconocidos como el colectivo mayoritario, por lo que son necesarias más investigaciones.

Algunas entidades han señalado que, la aporofobia, cuyo significado es el odio al pobre, es una de las motivaciones en el ejercicio de la violencia que sufren las personas sin hogar, cuyo énfasis, en la ciudad de São Paulo, se produce en su vertiente institucional. Por lo tanto, la exposición de esta dinámica, unida a la escasez de estadísticas en la metrópoli mencionada, fueron el aliciente para el desarrollo de una investigación más profunda, la cual se encuentra, actualmente, en fase de análisis de datos, a pesar de que la conclusión anticipada coincide con lo señalado al inicio del párrafo. 
En síntesis, la idea fundamental que se deriva de la investigación efectuada es la necesidad de emprender una lucha contra la criminalización y victimización penal que se está produciendo en las personas sin hogar de la ciudad de São Paulo. No obstante, conviene destacar las dificultades que existen, como la contribución en este proceso del Estado brasileño a través de la adopción de políticas que favorecen su marginalización, estigmatización, e inclusive, el desarrollo de crímenes relacionados con sus situaciones de pobreza. De igual forma, una problemática fundamental consiste en la victimización del colectivo de sinhogarismo por los propios agentes que deben evitar la comisión de delitos en su persona, dicho de otro modo, las fuerzas policiales están ejerciendo violencia sobre las personas sin hogar, correspondiéndose estas con diferentes tipologías delictivas.

Finalmente, cabe resaltar que este trabajo también se configura como un reclamo al gobierno brasileño por la necesidad de protección del colectivo de sinhogarismo, con la siguiente paradoja ¿Quién va a ejercer esa función si es el propio Estado y sus operadores los autores de la criminalización y victimización que sufren? 


\section{NOTAS}

${ }^{1}$ En Brasil, la criminalización de la pobreza puede ser observada en documentos oficiales desde finales del siglo XX, marcada por una herencia de más de 300 años de esclavitud (Bouças, 2006).

${ }^{2}$ Esta tesis, cuyo máximo exponente es Günther Jakobs, ha tenido una amplia reproducción en los sistemas penales latinoaméricanos. Su idea principal es la diferenciación de un derecho penal del ciudadano, para los sujetos que cumplen esta condición, y un derecho penal del enemigo, para los que son categorizados como enemigos del sistema. Con relación a lo expuesto, defiende una relativización y disminución de las garantías penales para los individuos que son considerados como enemigos del sistema. En aplicación de esta tesis al Estado brasileño, el enemigo es caracterizado por ser negro y pobre, con especial énfasis a las personas sin hogar (Brandão, 2019).

${ }^{3}$ Término que hace referencia al grado de seguridad de las personas en su vida cotidiana.

${ }^{4}$ También resulta adecuada la definición otorgada por la policía de Brasil, definiendo a este colectivo como: "grupo populacional heterogêneo, caracterizado por sua condição de pobreza extrema, pela interrupção e fragilidade dos vínculos familiares e pela falta de moradia convencional regular" (Decreto $n^{\circ} 7053,2009$ ).

${ }^{5}$ En este periodo no se contabilizó la diferenciación del número de personas que pernoctaban en la calle y quienes se encontraban en albergues.

${ }^{6}$ Según datos del último censo realizado.

7 La conceptualización se produjo por Adela Cortina, proveniente de los vocablos áporos y phobia, cuya inspiración se derivó de la palabra xenofobia (Cortina, 2017).

${ }^{8}$ A través del estudio de la criminalización de la pobreza y la violencia ejercida por las fuerzas policiales en las detenciones efectuadas a las personas sin hogar.

9 En esta organización se desarrollaron entrevistas a personas sin hogar focalizadas en la victimización aporófoba. 


\section{REFERENCIAS BIBLIOGRÁFICAS}

Aguirre, R. T. (2018). O modelo penal da aporofobia. In A. B. Lopes, E. S. Fonseca, J. V. Meirelles, L. Pachani, \& S. Abid (Orgs). Direito penal, processo penal, execução penal e criminologia nos 30 anos de constituição cidadã: novos caminhos e desafios (pp. 445-461). Belo Horizonte, Brasil: D’Placido.

Bertti, R., \& Rodrigues, T. (2018). O direito à imagem dos indivíduos em situação de rua: voyeurismo da miséria, intimidade pública e o filtro da responsabilidade. In T. Rodrigues., \& V. S. Galdino (Eds.). Pessoas em situação de rua. Invisibilidade, Preconceitos e Direitos (pp.101112). Brasilia, Brasil: Zakarewicz Editora.

Bouças, C. M. (2006). Direitos Humanos e Criminalização da Pobreza. I Seminário Internacional de Direitos Humanos, Violência e Pobreza: a situação de crianças e adolescentes na América Latina hoje, Universidade do Estado do Rio de Janeiro, Brasil.

Brandão, Q. (2019). A seletividade do sistema penal no estado democrático brasileiro: a população negra, um direito penal do inimigo e a cidadania mínima - o caso Rafael Braga. In L. Chinchilla (Coord). Democracia, Liderança e Cidadania na América Latina (pp. 291-312). São Paulo: Editora da Universidade de São Paulo.

Carvalho, M. A. (2016). Estimativa da população em situação de rua no Brasil, Instituto de Pesquisa Econômica Aplicada (Ipea). Recuperado de [http://repositorio.ipea. gov.br/bitstream/11058/7289/1/td_2246.pdf].

Centurião, L. R., Rodrígues, T., \& Teixeira, L. (2018). Aluguel Social: Direitos e Desafios. In T. Rodrigues., \& V. S. Galdino (Eds.). Pessoas em situação de rua. Invisibilidade, Preconceitos e Direitos (pp. 241-256). Brasilia, Brasil: Zakarewicz Editora.

Cifali, A. C. (2016). Política criminal e governos de esquerda na América Latina. Entre semelhanças e ambiguidades. Civitas, 16(4), 673-681. Recuperado de [http://revistaseletronicas.pucrs.br/ojs/index.php/ civitas/article/view/24401].

Cortina, A. (2017). Aporofobia: el rechazo al pobre: un desafío para la sociedad democrática. Valencia: Paidós.

Decreto $n^{\circ}$ 7053. Presidência da República Casa Civil, Brasil, 23 de diciembre del 2009.

García, I. (2019). Aporofobia: una investigación cualitativa al colectivo de personas sin hogar en Salamanca. Ars luris Salmanticensis, 7, 25-53.

García, I. (2020). La aporofobia en el sistema penal español: especial referencia al colectivo de personas sin hogar. Salamanca: Ratio Legis.

Ghiringhelli, R., \& Cifali, C. (2016). Seguridad pública, política criminal y penalidad en brasil durante los gobiernos Lula y Dilma (2003-2014). Cambios y continuidades. In M. Sozzo (Ed). Postneoliberalismo y penalidad en América del Sur (pp. 29-94). Buenos Aires: CLACSO.

Kant de Lima, R. (1997). Polícia e exclusão na cultura judiciária. Tempo Social, 9(1), 169-183.

Kant de Lima, R., Misse, M., \& Mendes de Miranda, A. P. (2000). Violência, Criminalidade, Segurança Pública e Justiça Criminal no Brasil: uma bibliografia. Revista Brasileira de Informação Bibliográfica em Ciências Sociais, 50(2), 45-123.

Mello, D. (2018, 26). Esterilização de moradora de rua não é caso isolado, dizem entidades. Agência Brasil. Recuperado de [https://agenciabrasil.ebc.com. br/direitos-humanos/noticia/2018-06/esterilizacaode-moradora-de-rua-nao-e-caso-isolado-dizementidades]. Consultado [10-03-2020].

Ministério de Saúde (2019). População em situação de rua e violência - uma análise das notificações no Brasil de 2015 a 2017. Boletim epidemiológico, 50(14), 1-13.

Moura, J. (2018). O Direito penal do inimigo e a seletividade do sistema penal brasileiro no caso Rafael Braga. Monografia de conclusão de bacharelato, Faculdade de Ciências Jurídicas e Sociais, Brasília, Distrito Federal, Brasil.

Nascimento, N., Rodrígues, T., \& Centurião, L. F. (2018). Pessoas em situação de rua: aspectos sociais. In T. Rodrigues., \& V. S. Galdino (Eds.). Pessoas em situação de rua. Invisibilidade, Preconceitos e Direitos (pp. 81-89). Brasília: Zakarewicz Editora.

Observatorio Hatento. (2015). Muchas Preguntas, Algunas Respuestas. Recuperado de [http://hatento.org/ wp-content/uploads/2014/10/informe-diagnostico.pdf]. Consultado [10-03-2020].

Oliveira, V. (2018). Problemas dos Direitos Humanos das pessoas em situação de rua no Brasil. In T. Rodrigues., \& V. S. Galdino (Eds.). Pessoas em situação de rua. Invisibilidade, Preconceitos e Direitos (pp. 11-29). Brasília: Zakarewicz Editora.

Prefeitura Municipal de São Paulo (2015a). Pesquisa censitária da população em situação de rua, caracterização socioeconômica da população adulta em situação de rua e relatório temático de identificação das necessidades desta população na cidade de São Paulo. Relatório completo do censo da população em situação de rua na cidade de São Paulo. Produto V. Recuperado de [https://www.prefeitura.sp.gov.br/cidade/secretarias/ upload/00-publicacao_de_editais/0001.pdf]. Consultado [10-03-2020].

Prefeitura Municipal de São Paulo (2015b). Pesquisa censitária da população em situação de rua, caracterização socioeconômica da população adulta em situação de rua e relatório temático de identificação das necessidades desta população na cidade de São 
Paulo. Sumário executivo. Recuperado de [https:// www.prefeitura.sp.gov.br/cidade/secretarias/upload/00publicacao_de_editais/0003.pdf]. Consultado [15-032020].

Prefeitura Municipal de São Paulo (2015c). Pesquisa censitária da população em situação de rua, caracterização socioeconômica da população adulta em situação de rua e relatório temático de identificação das necessidades desta população na cidade de São Paulo. Produto IX. Relatório final da pesquisa amostral do perfil socioeconómico. Recuperado de [https:// www.prefeitura.sp.gov.br/cidade/secretarias/upload/00publicacao_de_editais/0004.pdf]. Consultado [15-032020].

Prefeitura Municipal de São Paulo (2015d). Pesquisa censitária da população em situação de rua, caracterização socioeconômica da população adulta em situação de rua e relatório temático de identificação das necessidades desta população na cidade de São Paulo. Produto XV. Complemento do Relatório final do censo e pesquisa amostral do perfil socioeconómico e de identificação das necessidades. Recuperado de [https://www.prefeitura.sp.gov.br/cidade/secretarias/ upload/00-publicacao_de_editais/0005.pdf]. Consultado [15-03-2020].

Prefeitura Municipal de São Paulo (2020a). Pesquisa Censitária da população em situação de rua. São Paulo SP, 2019. Recuperado de [https://app.powerbi.com/w?r =eyJrljoiYzM4MDJmNTAtNzhIMiOONzliLTk4MzYtY2MzN 2U5ZDE1Yzl3liwidCI6ImE0ZTA2MDVjLWUzOTUtNDZIY S1iMmE4LThINjE1NGM5MGUwNyJ9]. Consultado [1503-2020].

Prefeitura Municipal de São Paulo (2020b). Caracterização socioeconômica da população adulta em situação de rua e relatório temático de identificação das necessidades desta população na cidade de São Paulo - 2019, produto $\mathrm{v}$, relatório completo do censo. Recuperado de [file:///C:/ Users/Ana\%20Pérez\%20Cepeda/Desktop/Isabel/ Investigación\%20SP/Produto5_SMADS_SP_Final.pdf]. Consultado [15-03-2020].

Raupp, L. \& Adorno, R. (2010). Uso de crack na cidade de São Paulo/Brasil. Toxicodependencias, 16(2), 29-37.

Schramm, F. P. (2017). Violência contra população em situação de rua no Brasil é denunciada à ONU. Terra de Direitos. Recuperado de [https://terradedireitos.org. $\mathrm{br} /$ noticias/noticias/violencia-contra-populacao-emsituacao-de-rua-no-brasil-e-denunciada-a-onu/22629]. Consultado [15-03-2020].

Silvestre, G., Schilittler, M. C., \& Sinhoretto, J. (2016). Notas sobre as estratégias estatais de controle do crime em São Paulo. $39^{\circ}$ Encontro Anual da ANPOCS, SPG 05 - O lugar da prisão na sociedade contemporânea: dilemas e desafios, Minas Gerais, Brasil.

Sinhoretto, J., Schilittler, M. C., \& Silvestre, G. (2016). Juventude e violência policial no Município de São Paulo. Revista Brasileira de Segurança Pública, 10(1), 10-35.
Teixeira, C. (2016). Vulnerabilidade da população em situação de rua na cidade de são paulo, brasil: mitos e especificidades. XXI Congreso Internacional del CLAD sobre la Reforma del Estado y de la Administración Pública, Centro Latinoamericano de Administración para el Desarrollo (CLAD) y el Gobierno de Chile, Santiago, Chile.

Valente, F. (2018). Ministro Dias Toffoli nega HC a réu reincidente por furto de bermuda de $R \$ 10$. Recuperado de [https://www.conjur.com.br/2018-jun-30/toffoli-negahc-reu-reincidente-furto-bermuda-10]. Consultado [1503-2020].

Young, J. (2002). A sociedade excludente. Exclusão social, criminalidade e diferença na modernidade recente. Rio de Janeiro: Revan.

Zaffalon, L. (2018). A política da justiça. Blindar as elites, criminalizar os pobres. São Paulo: Hucitec. 